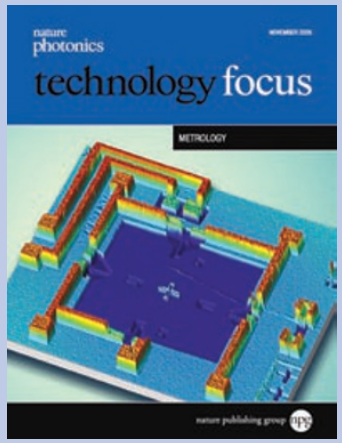

Cover image

Interferometry has become a valuable tool for measuring microelectromechanical systems.

Industry Perspective p661

EDITORS: NADYA ANSCOMBE, OLIVER GRAYDON

PRODUCTION EDITOR: CHRIS GILLOCH

COPYEDITOR: ANNA DEMMING

ART EDITOR: TOM WILSON

naturephoton@nature.com

\title{
Meeting demand
}

A 11 high-quality products, from aircraft engines to aspherical lenses or microchip circuits, need to be measured to check if they meet the customer's specifications. In many cases, optical metrology is a highly attractive solution owing to its resolution, speed and non-contact approach. Although other techniques, such as atomic-force and electron microscopy, are able to provide detailed information, the down side is that they require expensive equipment, the measurements are time-consuming and special preparation of the specimen is required. Optical metrology is often seen as a more practical and cost-effective approach. But today's products are placing ever increasing demands on metrology systems.

Minaturization, especially in the semiconductor industry, means that we can no longer 'see' the products we are trying to measure, and researchers are coming up with clever solutions to indirectly measure small feature sizes (page 672). Microelectromechanical-system (MEMS) devices are also becoming more challenging to measure (page 661).

In manufacturing, companies often want metrology systems that have high resolution (both spatial and temporal), but are robust enough to work in an industrial environment. Vibration and air turbulence can be a problem for most optical metrology systems, but one US company believes it has come up with a solution (page 664).

In-line optical metrology enables manufacturing companies to check all their products instead of a sample selection.

However, when aircraft-engine manufacturer GE wanted to implement optical metrology in its manufacturing facilities, it could not find the right technology on the market to fulfil all its requirements. So the company developed its own system (page 667).

This is a common problem, and the answer may lie with the development of highly flexible metrology systems that can be customized to suit many situations. Such systems do not yet exist, but with today's huge computing powers and clever optical technology, perhaps the solution is just around the corner.
RESEARCH HIGHLIGHTS

$659 \quad$ New tools for applications from astronomy to singlecell biomechanics

INDUSTRY PERSPECTIVE 661 Scanning interferometry: Measuring microscale devices Mike Conroy and Daniel Mansfield

664

Dynamic interferometry: Beating vibration Mike Zecchino

Industrial metrology: Engineering precision Kevin Harding

\section{BUSINESS NEWS}

670

An acquisition provides opportunity for growth, sales fluctuate, and more

\section{PRODUCT HIGHLIGHTS}

671 A large-area measurement system, noise-insensitive interferometers, and more

\section{INTERVIEW}

672

Measuring up to industry Interview with Wolfgang Osten 